\title{
Vertex Reconstruction in the ATLAS Experiment at the LHC
}

\author{
E. Bouhova-Thacker, T. Koffas, V. Kostyukhin , W. Liebig, M. Limper, G. Piacquadio, K. Prokofiev, \\ C. Weiser, A. Wildauer, on behalf of the ATLAS Collaboration.
}

\begin{abstract}
In the harsh environment of the Large Hadron Collider at CERN (design luminosity of $10^{34} \mathbf{c m}^{-2} \mathbf{s}^{-1}$ ) efficient reconstruction of vertices is crucial for many physics analyses. Described in this paper are the strategies for vertex reconstruction used in the ATLAS experiment and their implementation in the software framework Athena. The algorithms for the reconstruction of primary and secondary vertices as well as for finding of photon conversions and vertex reconstruction in jets are described. A special emphasis is made on the vertex fitting with application of additional constraints. The implementation of mentioned algorithms follows a very modular design based on object-oriented $\mathrm{C}++$ and use of abstract interfaces. The userfriendly concept allows event reconstruction and physics analyses to compare and optimize their choice among different vertex reconstruction strategies. The performance of implemented algorithms has been studied on a variety of Monte Carlo samples and results are presented.
\end{abstract}

\section{INTRODUCTION}

87 HIS paper describes vertex reconstruction in the ATLAS Experiment at the Large Hadron Collider (LHC) $\delta^{b}$ at the European Organization for Nuclear Research (CERN), Theneva, Switzerland. It presents the modular design of the uf software for vertex reconstruction and properties of its con染ete applications. The performance is demonstrated on several هidrtex topologies such as primary vertex finding, reconstrucr t i 官解ing and vertex finding in jets.

\section{The ATLAS Detector}

3 The ATLAS Detector [1] is a particle detector in operation 尊 2 the LHC at CERN. It is a multi-purpose detector designed to cover a wide range of physics analyses. For vertex reconstruction, the Inner Detector (ID) is of most importance. The ATLAS ID consists of three subsystems which, from inside to outside, are

1 three barrel and $2 \times 3$ end-cap silicon pixel layers with an $\mathrm{R} \phi$ resolution of $12 \mu \mathrm{m}$

Manuscript received November 14, 2008. W. Liebig has been supported by the Netherlands Organization for Scientific Research (NWO), research grant VIDI 680.47.218.

E. Bouhova-Thacker is with Lancaster University, Lancaster LA1 4YB, UK.

V. Kostyukhin is with INFN, Via Dodecaneso 33, 16146 Genova, Italy.

W. Liebig, M. Limper are with Nikhef, Science Park 105, 1098 XG Amsterdam, Netherlands.

G. Piacquadio, C. Weiser are with Physikalisches Institut, Albert-LudwigsUniversität Freiburg, Hermann-Herder-Str. 3, 79104 Freiburg, Germany.

T. Koffas, K. Prokofiev, A. Wildauer are with CERN, 1211 Genève 23, Switzerland

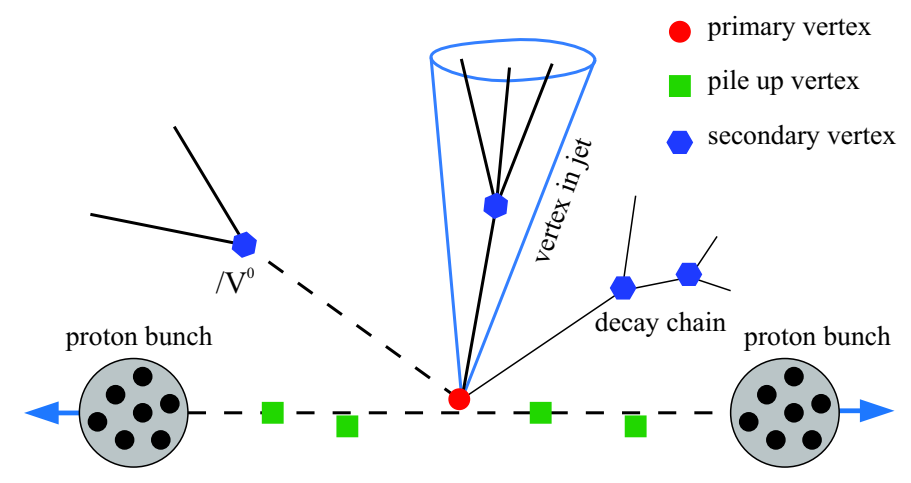

Fig. 1. Vertex topologies important for physics analyses in ATLAS: primary and pile up vertices, vertices from conversions and long-lived particles, vertices in jets and vertices from decay chains.

2 four barrel and $2 \times 9$ end-cap silicon microstrip layers with an $\mathrm{R} \phi$ resolution of $16 \mu \mathrm{m}$

3 transition radiation tracker with $\mathrm{e}^{ \pm}$identification and an $\mathrm{R} \phi$ resolution of approximately $170 \mu \mathrm{m}$

It provides on average $3+4+36$ measurements per charged particle trajectory, thus allowing for efficient reconstruction of tracks and vertices.

\section{VERTEX TOPOLOGIES}

The collision of the two proton bunches in the LHC with a center-of-mass energy of $14 \mathrm{TeV}$ leads to several different vertex topologies as shown in Fig. (1). In a typical collision event, several primary vertices along the beam as well as decays of long-lived particles, photon conversions, vertices in jets and vertices from decay chains, are produced. The reconstruction of vertices from these processes, distinguished by their different topologies, forms a vital part of the data analysis.

The reconstruction of primary vertices is important for many physics studies, including searches for new particles, tagging of $\mathrm{b}$ - and $\tau$-jets, reconstruction of exclusive b-decays and similar applications. The correct identification of the primary vertex which stems from the hard inelastic collision among many low- $\mathrm{p}_{\mathrm{T}}$ proton-proton interactions (so called minimum bias events) is of particular importance in the LHC conditions.

The high accuracy of the silicon tracker will allow ATLAS to select jets from b-quarks by searching for tracks originating from a separate b-hadron decay vertex in the vicinity of the primary vertex. The efficient detection and reconstruction of such displaced vertices is actually essential to achieve a good 
b-tagging performance. However, the fragmentation of $a$ quark results in a decay chain composed of a secondary vertex from the weakly decaying b-hadron and typically one or more tertiary vertices from c-hadron decays. The limited experimental resolution and high track density inside a jet therefore demand a dedicated vertex reconstruction, for which several approaches exist in ATLAS.

Particle decays in flight and even full decay chains are reconstructed using dedicated vertex finders. They exploit the assumed properties of the virtual particle and the conservation laws governing its decay, and use them to apply additional kinematic constraints in the vertex fit. About $40 \%$ of all photons produced in $p p$ collisions in ATLAS will convert in the material of the ID into $\mathrm{e}^{+} \mathrm{e}^{-}$pairs [1]. Hence, reconstruction of conversions is important for many physics analyses with photons in their final states. Reconstruction of conversions is also important to study the distribution of material in the detector (e.g. detector description in simulation, calibration of calorimeters). Finally the decays of long-lived neutral particles $\left(\mathrm{V}^{0}\right.$ 's) are determined. This is useful for instance for b-tagging where tracks from $\mathrm{V}^{0}$ 's degrade the b-tagging performance due to their relatively large impact parameters. As in the case of secondary vertex reconstruction, these applications employ constrained vertex fitting.

\section{SOFTWARE FRAMEWORK}

The aim of the vertex reconstruction software in ATLAS is to provide a common and modular infrastructure to reconstruct different vertex topologies discussed in the previous section. This is achieved by using a common Event Data Model and common abstract interfaces based on object oriented $\mathrm{C}++$. The software for vertex reconstruction is fully integrated into the general ATLAS computing environment [2].

\section{A. Event Data Model}

The Event Data Model (EDM) defines the data classes in which information relevant for vertex reconstruction is stored. It is also used to transfer information between algorithms and to store final results on disk. The EDM consists of classes representing reconstructed vertices and their relation to other objects used during a vertex reconstruction process (tracks, jets, etc.). Different vertex topologies also require different levels of detail concerning the data which needs to be stored in the various EDM classes. This is achieved by using inheritance throughout the data model. This approach has two important advantages:

1 Quantities which are common to all vertex topologies are stored and retrieved the same way (common look and feel for users). This includes e.g. the vertex position and the vertex-track relations.

2 Only the required amount of detail for a given vertex topology is stored in the objects and hence on disk (efficient use of disk space is of high importance in ATLAS).

The EDM for vertex reconstruction also uses data classes of the general EDM for reconstruction of tracks whenever it has to deal with tracking quantities like track parameters or

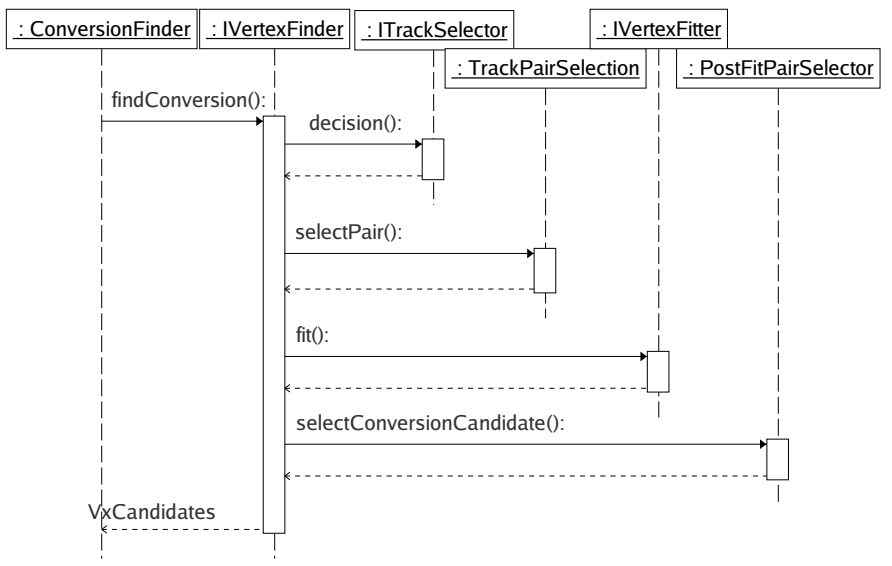

Fig. 2. Example sequence diagram showing the usage of abstract interfaces to define the reconstruction of conversions $\left(\gamma \rightarrow \mathrm{e}^{+} \mathrm{e}^{-}\right)$.

error matrices. End users and developers alike profit from this sharing of data classes.

\section{B. Interfaces}

Next to a common event data model, abstract interfaces are defined for all vertex reconstruction and related helper tasks. Algorithms for vertex reconstruction such as primary vertex finding or reconstruction of conversions make use of these interfaces to complete different steps of their reconstruction chain. Each interface can have several concrete implementations. The implementation used at each step is defined during run time by a steering file.

An excerpt of various interfaces for vertex reconstruction is presented as follows:

1 IVertexFinder: An interface for the implementation of vertex finders. Concrete implementations analyze a track collection and return a set of reconstructed vertices (i.e. a vector of primary and minimum bias vertices).

2 IVertexFitter: An interface for the implementation of vertex fitters. The fit method operates on a set of reconstructed tracks and, optionally, on a starting point for the fit. An additional vertex constraint (typically the beam spot) can also be used. The interface returns a single reconstructed vertex.

3 IVertexSeedFinder: An interface for the algorithms estimating the starting point of the vertex fit, given a set of tracks.

4 IVertexLinearizedTrackFactory: An interface for storing the parameters of the linearized measurement equation, representing the dependence of the track parameters on the vertex position and on the track momentum at the vertex.

5 IVertexUpdator: An interface for tools which are called iteratively to update the vertex estimate with one track at a time. Concrete implementations allow the addition or removal of a single track to or from a vertex candidate.

6 IVertexSmoother: An interface for the implementation of algorithms which update the parameters of all tracks fitted to a vertex with the knowledge of the vertex position. 
7 ISecVertexInJetFinder: An interface for the reconstruction of secondary vertices in jets. As an input, the reconstructed primary vertex, the tracks associated to a jet and the jet direction as reconstructed in the calorimeter are provided, while the expected output is a set of one or more reconstructed displaced vertices.

8 IVertexKinematicFitter: An interface for fitting a set of identified particles, i.e. tracks with associated particle masses, to a common vertex using a variable list of constraints.

9 IKinematicConstraint: An interface for providing vertex fitters with additional constraint equations. When invoked, each implementation supplies values and derivatives for a set of identified particles.

A simple use-case of these interfaces to define for example the reconstruction of conversions is given in Fig. (2). The so called ConversionFinder algorithm uses (amongst others) concrete implementations of the IVertexFinder and IVertexFitter interfaces. The former object is responsible to find conversion candidates. It applies pre-selection cuts to a set of input tracks and retains pairs of oppositely charged tracks which are likely to originate from photon conversions. The latter object performs the actual vertex fit of conversion candidates and returns the fitted vertex. This procedure is often done in a loop where the actual fit gives feedback to the finder concerning the "quality" of the vertex fit. The finder then decides to go on or to accept the fitted vertex.

This structure of abstract interfaces, in conjunction with an event data model for vertex reconstruction, provides a common look and feel to the end-user who, regardless of the vertex topology, always works with the same EDM classes and interfaces. This design also allows for a high level of modularity and flexibility. Concrete implementations can be exchanged or added easily by modifying the external steering, without the need to update client software. In addition, many different approaches can be applied and tested in parallel to achieve the best result for a certain vertex topology.

In the following sections, several vertex reconstruction chains which are based on these interfaces and event data model are presented and preliminary results are shown.

\section{Reconstruction of Primary Vertices}

During the initial years, the Large Hadron Collider will operate at a luminosity of $2 \cdot 10^{33} \mathrm{~cm}^{-2} \mathrm{~s}^{-1}$ (low luminosity), reaching $10^{34} \mathrm{~cm}^{-2} \mathrm{~s}^{-1}$ (design luminosity) at later stages. The average number of proton-proton interactions per bunch crossing is distributed according to a Poisson distribution with averages of 4.6 and 24 for these two luminosity modes, respectively. Each signal event reconstructed in the ATLAS detector will thus be superimposed with several minimum bias events. Compared to the signal events, the minimum bias collisions usually have lower track multiplicity and lower transverse momentum.

The identification of the signal primary vertex as well as precise reconstruction of its position is crucial for investigations of physics processes such as $H \rightarrow 4 l, H \rightarrow \gamma \gamma$, identification of $\mathbf{b}$ - and $\tau$-jets, reconstruction of exclusive $\mathbf{b}$-decays
TABLE I

EFFICIENCIES (IN \%) OF RECONSTRUCTION OF SIGNAL PRIMARY VERTICES WITH DIFFERENT APPROACHES.

\begin{tabular}{|c||c||c||c||c|}
\hline$\%$ & AMVF & VKalVrt & Fast fitter & Full fitter \\
\hline$W H, H(120) \rightarrow b \bar{b}$ & 95.35 & 96.25 & 89.38 & 89.39 \\
\hline
\end{tabular}

TABLE II

RESOLUTIONS ON THE TRANSVERSE POSITIONS OF SIGNAL PRIMARY VERTICES RECONSTRUCTED USING DIFFERENT APPROACHES.

\begin{tabular}{|c||c||c||c||c|}
\hline$[\mu \mathrm{m}]$ & AMVF & VKalVrt & Fast fitter & Full fitter \\
\hline$W H, H(120) \rightarrow b \bar{b}$ & 11.21 & 11.34 & 12.69 & 12.69 \\
$W H, H(120) \rightarrow u \bar{u}$ & 10.02 & 10.03 & 10.68 & 10.68 \\
$H \rightarrow 4 l$ & 9.81 & 9.72 & 10.4 & 10.4 \\
$t \bar{t}$ & 10.33 & 10.33 & 12.55 & 12.55 \\
\hline
\end{tabular}

and similar studies. The reconstruction of primary vertices can generally be subdivided in two stages: primary vertex finding, dealing with the association of tracks to a particular vertex candidate and vertex fitting, responsible for the reconstruction of the vertex position and corresponding covariance matrix. It is evident that since often these two processes are not easily distinguishable from each other, the "finding-throughfitting" and "fitting-after-finding" approaches are possible. The creation of separate base classes for algorithms responsible for vertex finding and fitting (Sec. IV-B) allow for modular implementation of both approaches in ATLAS. In addition, this modular approach allows for an easy exchange of tools between algorithms, adopting them to the properties of physics processes under study and using the best combination to yield the best result.

The Adaptive Multi Vertex Fitter (AMVF) is currently the default strategy for primary vertex finding in ATLAS. It is an example of the "finding-through-fitting" approach. The reconstruction starts with selection of tracks which are likely to originate from the interaction region. A single primary vertex candidate including all selected tracks is then formed and fitted with an Adaptive Multi Vertex Fitter. The tracks which are considered to be outliers during the first iteration of the fit are used to create a new vertex seed. The simultaneous adaptive fit of two vertices is then performed. The number of vertex candidates grows in each iteration and these candidates compete with each other in order to gain more tracks. The annealing procedure prevents the finding procedure from falling into local minima.

An example of the "fitting-after-finding" approach in ATLAS is the InDetPriVxFinder algorithm. Here the reconstructed tracks compatible with the interaction region are preselected and the primary vertex candidates are formed by searching for clusters of tracks in the longitudinal projection. These clusters are then iteratively fitted with one available vertex fitter, rejecting the outliers at every iteration. The maximal number of reconstructed vertices is thus fully determined at the seeding stage. Once a track is rejected from a vertex candidate, it is never used for any other cluster.

The variety of vertex fitters which can be used with InDetPriVxFinder includes the fast and full versions of the fitter proposed by P .Billoir [3], denoted hereafter as Fast and Full 

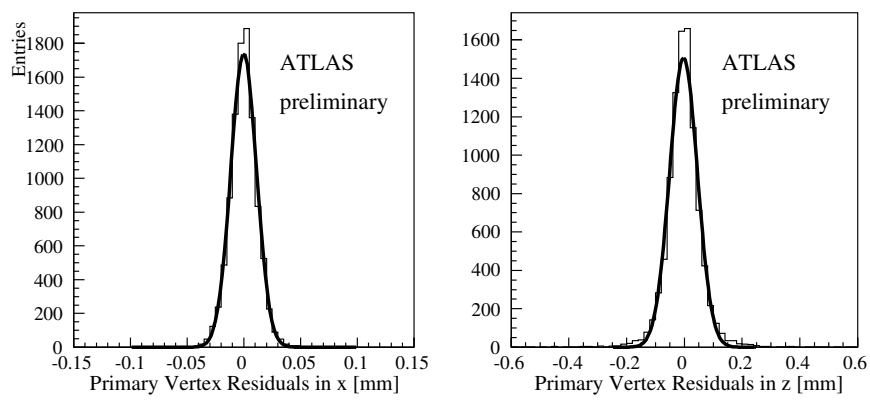

Fig. 3. Distribution of residuals of the transverse position of primary vertices in $t \bar{t}$ events reconstructed with the Adaptive Multi Vertex Finder.

fitters and a similar approach implemented in the VKalVrt package [4].

Presented in Table I are the efficiencies of reconstruction of signal primary vertices in the $W H, H\left(M_{H}=120 G e V\right) \rightarrow$ $b \bar{b}$ channel obtained by using different approaches currently implemented in ATLAS.

The statistical errors are below $1 \%$ in all the cases. It can be noted that the highest efficiencies are provided by the Adaptive Multi Vertex Finder and VKalVrt algorithms. Indeed, comparing to the other finders, these methods provide more robust approaches allowing the number of vertex candidates to be changed during the finding process and to deal efficiently with outlying tracks

Presented in Table II are the resolutions on the x-coordinate of signal primary vertices reconstructed with different approaches. The study is performed on a set of Monte Carlo samples, simulating the $W H, H(120) \rightarrow b \bar{b}$, the $W H, H(120) \rightarrow$ $u \bar{u}$, the $H \rightarrow 4 l$ and the $t \bar{t}$ channels in the low luminosity conditions. It can be noted that all the resolutions on transverse positions of reconstructed vertices are similar in all approaches: approximately $10 \mu \mathrm{m}$ to $12 \mu \mathrm{m}$, depending on the approach and the kinematics of the channel. The calculated errors on the resolution values are typically smaller than $1 \mu \mathrm{m}$.

The resolutions on the longitudinal positions on primary vertices are typically of the order of $35 \mu \mathrm{m}$ to $55 \mu \mathrm{m}$, depending on the approach and the kinematics of the channel. Presented in Fig. (3) and Fig. (4) are the distributions of residuals and pulls of the transverse and longitudinal components of the primary vertex position of $t \bar{t}$ events reconstructed with the Adaptive Multi Vertex Finder. It can be noted that the distributions are reasonably well approximated by a Gaussian and that the errors on the reconstructed vertex positions are correctly estimated.

\section{Vi. Vertex Reconstruction with Kinematic CONSTRAINTS}

The reconstruction of a secondary vertex, whether this is due to a heavy flavour decay, converted photon or longlived hadron, usually involves the application of kinematic constraints in the vertex fit. Constrained vertex fitting is therefore used in several packages in the ATLAS software framework. Most commonly it is implemented under an "allin-one" fitting approach that carries specific applications of constraint vertex fitting out, such as the secondary vertex and
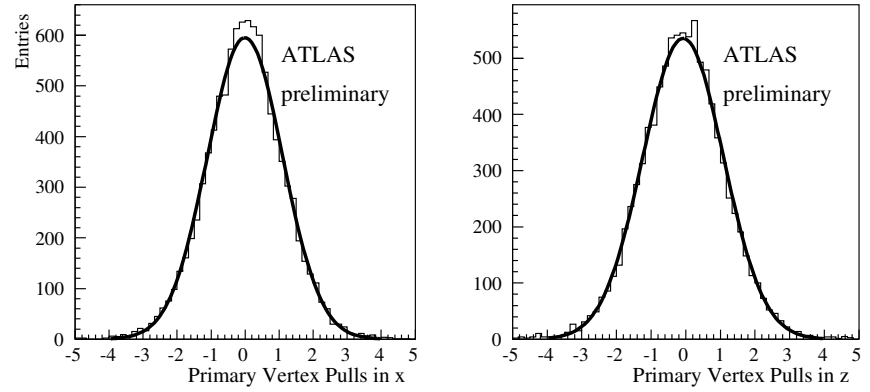

Fig. 4. Distribution of pulls of the transverse position of primary vertices in $t \bar{t}$ events reconstructed with the Adaptive Multi Vertex Finder.

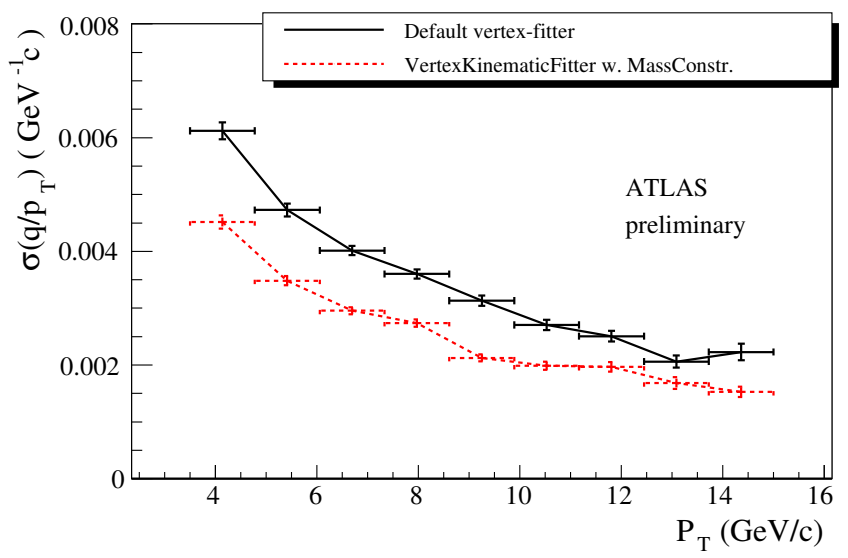

Fig. 5. Momentum resolution of the muon track parameters at the vertex reconstructed with the default vertex fitter and the VertexKinematicFitter with mass constraint, using simulated $J / \psi \rightarrow \mu^{+} \mu^{-}$events.

decay chain fitting in the VKalVrt package [4]. The most useful type of constraint forces the mass of the decaying particle to the value corresponding to the particle hypothesis. In the case of a converted photon, the massless nature of the decaying particle allows the mass constraint to be applied directly as a collinearity constraint on the track parameters, leading to improved robustness of the fit convergence.

Recently, a new tool for constrained vertex fitting has been developed under the modular concept described in Section (IV). This new tool, VertexKinematicFitter, is based on $\chi^{2}$ minimization with Lagrange multipliers [6], to simultaneously satisfy a vertex-constraint and any other kinematic constraint requested by the user. The Lagrange formalism allows each different constraint to be implemented as a separate class with a common abstract interface. An example of the use of the VertexKinematicFitter is given in Fig. (5), showing how the use of a mass-constraint in simulated $J / \psi \rightarrow \mu^{-} \mu^{+}$ events improves the resolution of the momentum measurement (curvature) of the two emerging muon tracks.

\section{Reconstruction of CONVERTED PhOtONS AND Long-Lived Neutral Particles}

The reconstruction of a long-lived particle decay or a converted photon vertex involves the application of a mass or angular constraint. The basic structure of constrained vertex reconstruction is very similar in both cases as Fig. (2) 


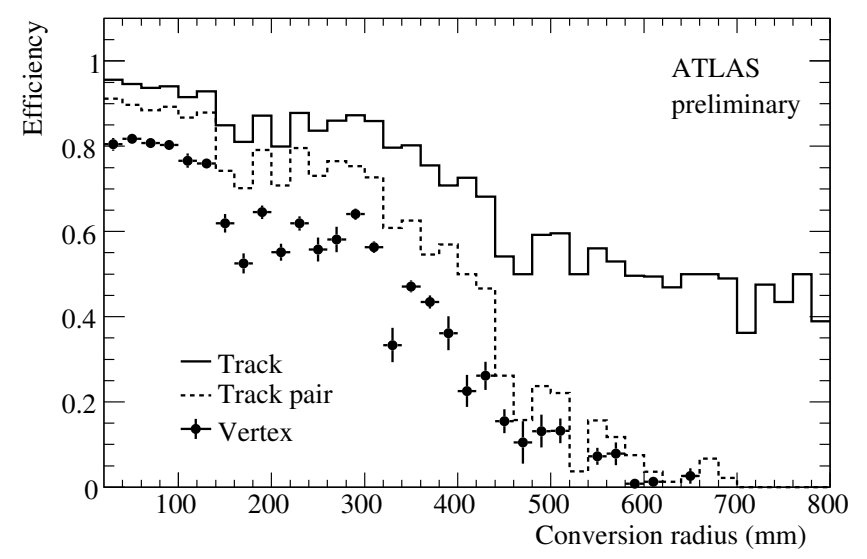

Fig. 6. Track, track-pair and vertex reconstruction efficiency for converted photons originating from $120 \mathrm{GeV} H \rightarrow \gamma \gamma$ decays, as a function of distance from the beam axis.

illustrates in the case of the photon conversion. Here the basic components are: the track selection and subsequent track classification, the formation of pairs of tracks with opposite charge, the vertex fitting and reconstruction of photon conversion vertex candidates, and the final post-fit selection of the conversion candidates. Constrained vertex fitting can be very CPU-time consuming, so a careful pre-selection of track and pair candidates is necessary. In the case of converted photons, for instance, electron tracks are selected using the particle identification capabilities provided by the ATLAS Transition Radiation Tracker (TRT). Track pairs are also preselected by requiring the two tracks of a converted photon to have small initial polar angle differences. In the case of both, converted photons and massive decaying particles, the distance of minimum approach between the two tracks in question has to be sufficiently small. In order to facilitate the convergence of the constrained fit, a reasonable initial estimate of the vertex position is also provided. All the above pre-selection results in a significant reduction of the combinatorial background before the actual constrained vertex fit is performed. Additional reduction is achieved by exploiting the vertex fit results themselves. In the case of long-lived decaying particles an additional step involving an un-constrained vertex fit is needed in order to determine the appropriate mass constraint to be applied.

Only tracks originating from a radial distance from the beam axis of up to approx. $800 \mathrm{~mm}$ can be efficiently reconstructed by the tracking algorithm. However, due to the more limited resolution of the ATLAS TRT, the efficiency for reconstructing track pairs is significantly reduced at radial distances above $400 \mathrm{~mm}$. This becomes even more pronounced in the case of high $\mathrm{p}_{\mathrm{T}}$ decaying particles. In addition, due to actual ATLAS tracker geometrical constraints, the track reconstruction efficiency is severely curtailed for pseudorapidity values of $|\eta|>$ 2 , although tracks are still reconstructed for up to $|\eta|=2.5$. This can be clearly seen in Fig. (6), where the reconstruction efficiencies for converted photons originating from a $120 \mathrm{GeV}$ $H \rightarrow \gamma \gamma$ decay are shown.

Fig. (7) shows the resolution of the reconstructed vertex

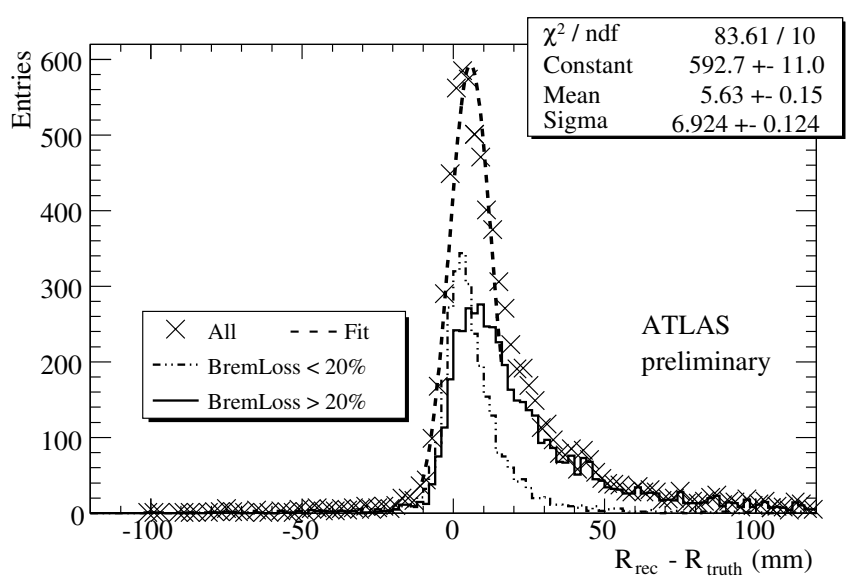

Fig. 7. Reconstructed vertex radial position resolution (in $\mathrm{mm}$ ) for converted photons from a $120 \mathrm{GeVH} \rightarrow \gamma \gamma$ decay. For comparison the two cases where the participating tracks have lost $>20 \%(<20 \%)$ of their energy due to bremsstrahlung are also shown separately.

radial position for the case of converted photons originating from $120 \mathrm{GeV}$ Higgs boson decays. The constrained vertex fit, requiring tracks to have the same direction at the vertex, has been used. The long tail to the right is due to bremsstrahlung losses of the two produced electron tracks, which reduce the individual track parameter reconstruction quality, hence severely affecting also the vertex fit results. To illustrate this particular point, the radial position resolution, with/without significant $(>20 \%$ ) losses due to bremsstrahlung, is also plotted separately. Clearly the tail is reduced when both contributing electrons have lost $<20 \%$ of their energy due to bremsstrahlung effects. For the moment no bremsstrahlung energy loss corrections are applied, although work is progressing well towards achieving that.

An overall radial position resolution of approx. $7 \mathrm{~mm}$ has been achieved. The fact that the photon is a massless particle, resulting in an extremely small angular opening of the emitted tracks, makes it more difficult to reconstruct accurately the position of the conversion vertex. Position resolution is of the highest importance, since the reconstructed converted photons can offer the means to accurately map the ATLAS tracker material using minimum bias data. For more details on the conversion reconstruction and their applications, one can look in [5].

Bremsstrahlung losses on the other hand are not present in the case of the $K_{S} \rightarrow \pi^{+} \pi^{-}$decay. This, as well as the non-zero opening angle, provide a better test scenario for the constrained vertex fitting. As mentioned above, instead of the angular constraint used in the case of the photon conversions, a direct mass constraint is implemented. Fig. (8) shows the resolution of the reconstructed radial position for $K_{S}$ decays in $B_{d} \rightarrow J / \psi K_{S}$ events. The absence of a bremsstrahlung related tail compared to that in Fig. (7) is evident. The overall radial position resolution is approx. $0.3 \mathrm{~mm}$. The variation of the radial position resolution with the distance from the beam axis is shown in Fig. (9) for the case of $K_{S}$ decays. The overall resolution degrades as we move to higher radial distances and thus to the lower precision ATLAS tracker components. 


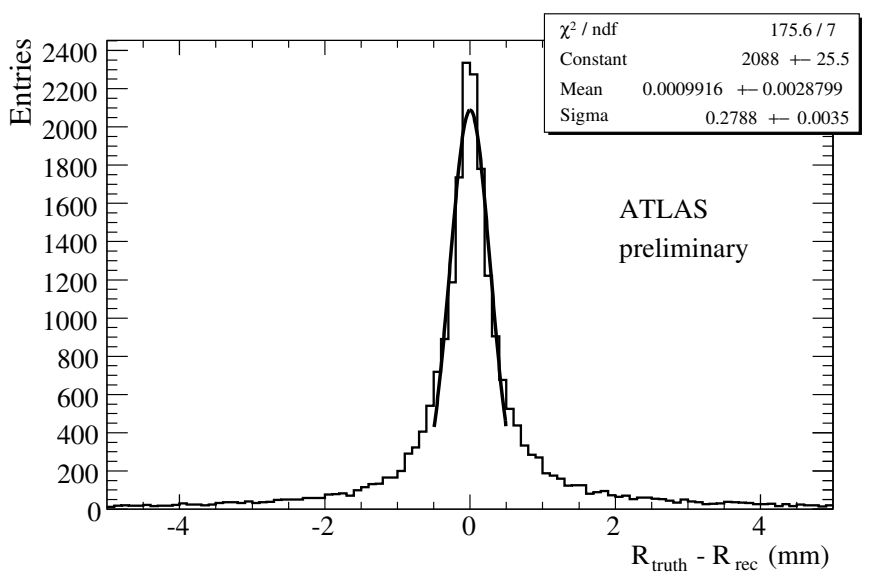

Fig. 8. Reconstructed vertex radial position resolution (in $\mathrm{mm}$ ) of $K_{S} \rightarrow$ $\pi^{+} \pi^{-}$decays.

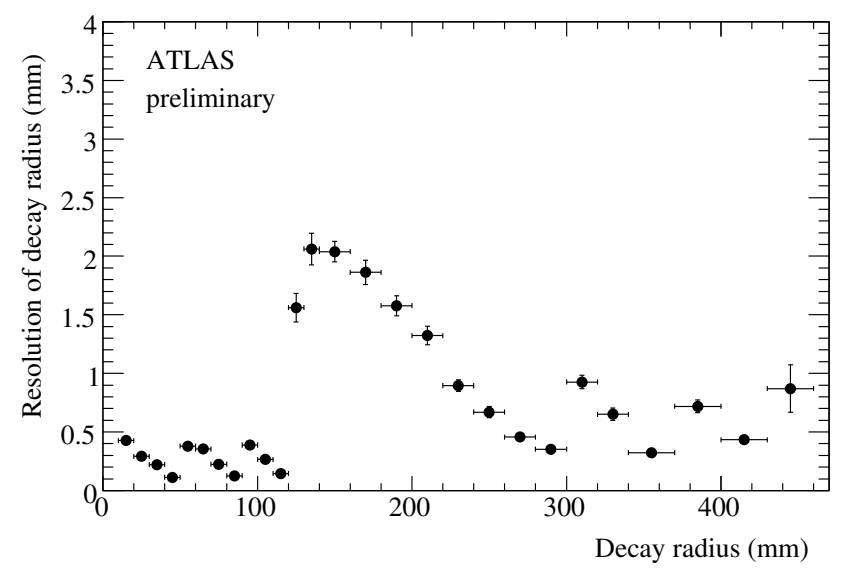

Fig. 9. Reconstructed vertex radial position resolution (in $\mathrm{mm}$ ) of $K_{S} \rightarrow$ $\pi^{+} \pi^{-}$decays as a function of the radial distance from the beam axis.

The large increase at approx. $115 \mathrm{~mm}$ is due to the 200 $\mathrm{mm}$ gap between the Pixel and the Silicon Strip Trackers. Similar behavior is exhibited by the conversion radial position resolution, albeit with a certain amount of deterioration due to the bremsstrahlung losses, as discussed above.

\section{RECONSTRUCTION OF VERTICES IN JETS}

The most basic b-tagging algorithms in ATLAS rely directly on the impact parameter significance of the charged particles associated to the jet, computed with respect to the primary vertex $(\mathrm{PV})$ reconstructed in the event. The detection and reconstruction of one or more secondary vertices in a b-jet can considerably improve the b-tagging performance, in two main respects:

- Identify real long-lived particles stemming from the fragmentation of u, d, s-quarks in light-quark jets into $K_{S}$, $\Lambda$ and conversions, thus avoiding their decay products to fake tracks coming from b-decays.

- Identify and exploit the production and kinematic properties of the b-decay and $b \rightarrow c$-decay vertices (fragmentation function, invariant mass, decay multiplicity) as
TABLE III

VERTEX RECONSTRUCTION EFFICIENCY IN B-,C- AND LIGHT-JETS

\begin{tabular}{|c||c||c||c||c|}
\hline 1 Algorithm & Topology & b & c & 1 \\
\hline Inclusive finder & Nothing & 13.5 & 68.0 & 93.3 \\
& 1 inclusive vertex & 76.5 & 32.0 & 6.7 \\
\hline Topological finder & Nothing & 13.7 & 51.3 & 79.7 \\
& 1 Single Track & 9.9 & 17.4 & 13.6 \\
& 2 Single Tracks & 4.5 & 2.6 & 1.0 \\
& 1 Single Vertex & 49.6 & 25.1 & 5.2 \\
& 1 Vertex + 1 Track & 15.9 & 3.1 & 0.4 \\
& 2 Vertices & 6.3 & 0.5 & 0.04 \\
\hline
\end{tabular}

an additional handle against badly reconstructed primary tracks in light-quark jets.

Two main vertex finding algorithms for b-tagging are implemented in ATLAS, one relying on the reconstruction of all displaced tracks into a common geometrical vertex (inclusive vertex finder) and the second relying on the identification of the $\mathrm{PV} \rightarrow \mathrm{b} \rightarrow \mathrm{c}$ decay chain topology (topological vertex finder). The inclusive vertex finder relies on a vertex fitter based on the Billoir method [3] as implemented in the VKalVrt package [4], while the topological vertex finder relies on an extension of the Kalman Filter formalism for vertex reconstruction developed in ATLAS, implemented in the JetFitter package [7].

The initial finding strategy is common for both finders:

- Selection of displaced tracks

- Reconstruction of all 2-track vertices

- Removal of vertices compatible with $\gamma, K_{S}$ and $\Lambda$.

The inclusive vertex finder then tries to fit the tracks surviving this selection into a common geometrical vertex. Tracks with a bad $\chi^{2}$ contribution are removed iteratively from the fit, until the overall $\chi^{2}$ is below a predefined threshold. The vertex $\operatorname{Prob}\left(\chi^{2}\right)$ is not expected to follow a flat distribution, because of the assumption of a single geometrical vertex, so some tuning of this threshold to achieve the best efficiency to purity ratio is needed.

The topological vertex finder solves the pattern recognition problem relying on the assumption that all tracks intersect a common $\mathrm{PV} \rightarrow$ b-hadron $\rightarrow \mathrm{c}$-hadron flight axis, thus reducing a three-dimensional clustering problem down to a onedimensional problem:

- A first fit is performed, initializing the b-flight axis with the calorimetric jet direction and assuming that all tracks form single vertices: this determines the b-flight axis direction and its intersections with the single tracks.

- An iterative clustering procedure is performed: compatible vertices (with the primary vertex being one of them) are clustered two-by-two, in decreasing order of compatibility, resulting in the end in a well defined topology.

Tables III and IV show how the two different strategies perform on jets selected from a sample of $W H, H(120) \rightarrow b \bar{b}$, $c \bar{c}$ or $l \bar{l}$ Monte Carlo events. The first table shows the vertex reconstruction efficiency for jets of transverse momenta between 50 and $80 \mathrm{GeV}$ : in b-jets both algorithms reconstruct one or 
TABLE IV

TRACK-TO-VERTEX ASSOCIATION EFFICIENCY AND PURITY

\begin{tabular}{|c||c||c||c|}
\hline Finding algo. & Topology & Efficiency & Purity \\
\hline Inclusive & 1 inclusive B/D vertex & $69 \%$ & $92 \%$ \\
\hline Topological & 1 vertex & $74 \%$ & $91 \%$ \\
& 1 vertex +1 track & $80 \%$ & $85 \%$ \\
& 2 vertices & $85 \%$ & $89 \%$ \\
\hline
\end{tabular}

more vertices in around $70-75 \%$ of cases. In the case that a vertex is found, the inclusive vertex finder correctly associates to the found vertex $69 \%$ of all well-reconstructed tracks from a heavy flavour decay, keeping a very high purity (92\% of the tracks at vertex come really from $b-$ or $b \rightarrow c$ decays). The topological vertex finder, which doesn't rely on the hypothesis of a single geometrical vertex, correctly associates to the fitted decay chain a larger fraction of the reconstructed tracks stemming from a real b- or c-hadron decay, in particular when a topology beyond the single vertex one has been identified, at the cost of a slightly reduced purity.

Fig. (10) shows the resolution achieved on the inclusive bhadron decay vertex with respect to the true b-hadron position in the transverse plane (in the direction of flight of the bhadron). The core resolution is around $400 \mu m$, with a big tail due to the contribution of tracks from c-hadron decays.

The output provided by the vertex finder in form of reconstructed vertices is then used to compute the variables used by the secondary vertex based b-tagging algorithms:

- Fraction of energy from charged particles at the decay vertex/vertices

- Invariant mass at the vertex/vertices

- Multiplicity of the decay vertex/vertices

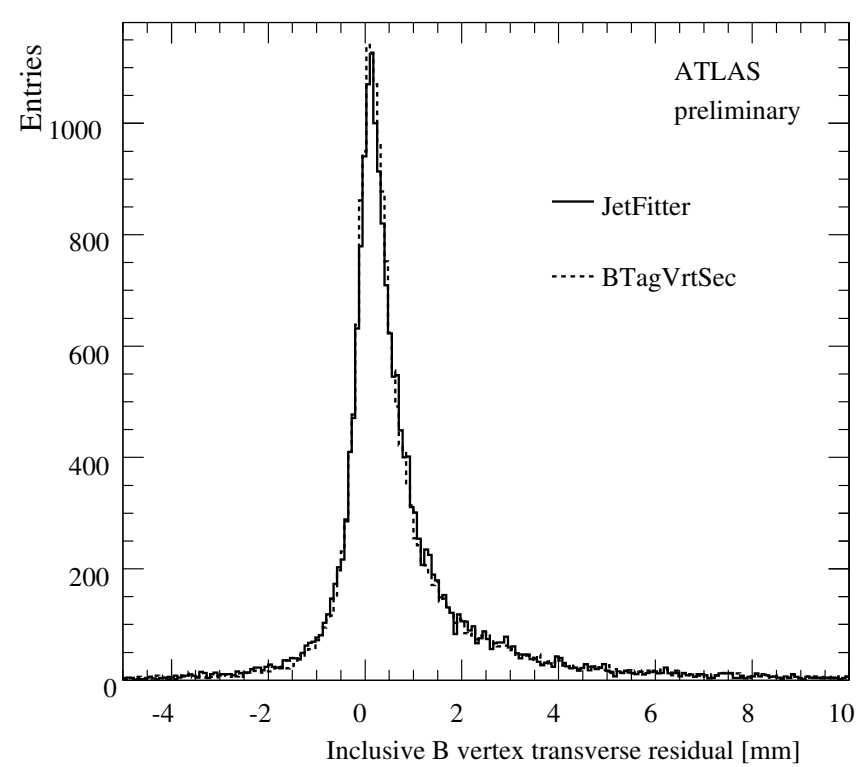

Fig. 10. Radial position resolution (in $\mathrm{mm}$ ) of the secondary vertex, for the inclusive vertex finder (BTagVrtSec in the figure) and for the topological vertex finder (JetFitter in the figure). In the latter case, only the first vertex in the decay chain is considered if more than one displaced vertex is found.
In addition, the information, whether a vertex was found or not (inclusive finder) or the identified decay chain topology (topological finder) is also used. This information is combined into a likelihood function. This is mostly a projective likelihood; however in the case of the inclusive finder, the correlations between the invariant mass and the fraction of energy from charged particles at the decay vertex is taken into account, using a two-dimensional probability density function $P D F$. The templates for the PDFs in the likelihood function are obtained from a very large number of Monte Carlo events, including $t \bar{t}, t \bar{t} j j$ and $W H$ events. Both secondary vertex based algorithms are then combined with the pure impact parameter based one, assuming that the two likelihood functions are uncorrelated.

The b-tagging performance achieved on $t \bar{t}$ and $t \bar{t} j j$ Monte Carlo events by the two secondary vertex based algorithms is illustrated in Fig. (11), where they are compared with the impact parameter only based algorithm. The rejection of light-quark jets is defined as the inverse of the probability to (mis)identify a light-jet as a b-jet. The plot shows that using the secondary vertex based algorithms implemented in ATLAS, it is possible to obtain a consistent improvement in the lightquark rejection for all b-tagging efficiencies below approx. $85 \%$.

\section{SUMMARY}

Presented in this paper is the software framework for vertex reconstruction in the ATLAS Experiment at the Large Hadron Collider at CERN. An overview of primary vertex reconstruction, reconstruction of conversions and long-lived particles, constrained vertex fitting and vertex finding in jets

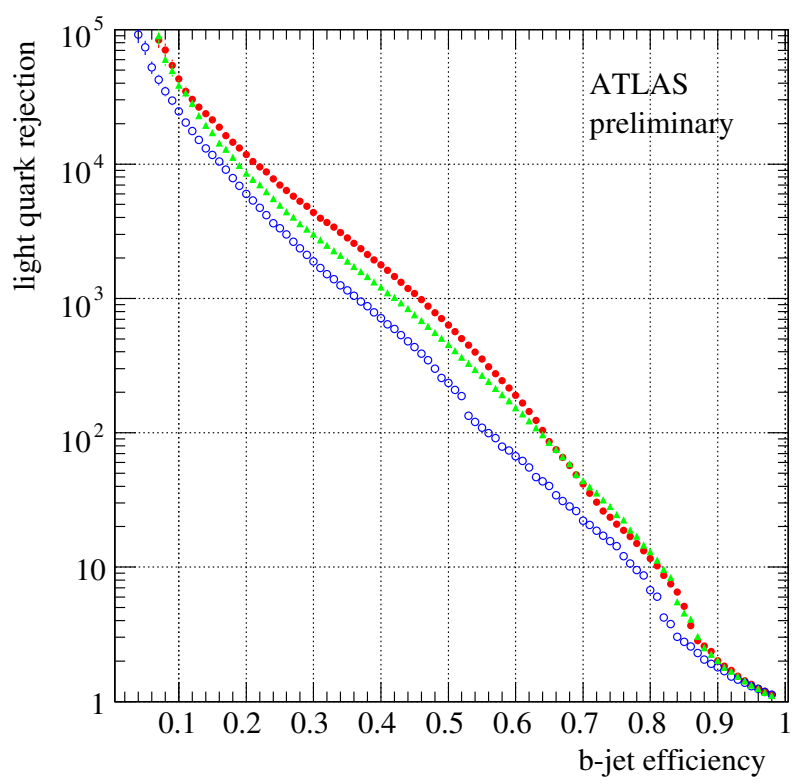

Fig. 11. Light quark rejection as a function of b-tagging efficiency for various algorithms: the inclusive vertex finder (green triangles), the topological vertex finder (full red circles) and, for comparison, the impact parameter only based algorithm (blue empty circles). 
for b-tagging is given. All these vertex topologies are handled by one single modular software environment. It defines a common Event Data Model and common abstract interfaces which are shared by all vertex reconstruction algorithms.

This vertex reconstruction software has been tested extensively under as real as possible conditions using the latest Monte Carlo datasets. The simulation of these data sets includes overlaid minimum bias vertices to account for the presence of pile up, a displaced proton-proton collision point, detector misalignment and more. The vertex algorithms perform satisfactory under these conditions and the ATLAS vertex community is eagerly awaiting to analyze real data from proton-proton collision in the LHC which are foreseen for next year.

\section{ACKNOWLEDGMENT}

The authors would like to thank the ATLAS Inner Detector community who helped developing and testing the vertex reconstruction software. In particular we would like to thank K. Grybel (Universitaet Siegen, Germany) for his help in producing some of the simulation data sets and $\mathrm{H}$. Zhu (University of Sheffield, UK) for his contribution to Fig. (7).

\section{REFERENCES}

[1] The ATLAS Collaboration, G. Aad et al., The ATLAS Experiment at the CERN Large Hadron Collider, JINST 3 (2008) S08003.

[2] The ATLAS Collaboration, ATLAS Computing Technical Design Report, ATLAS TDR-017, CERN-LHCC-2005-022.

[3] P. Billoir and S. Qian, Nucl. Instr. and Meth. A311 (1992) 139-150.

[4] V. Kostyukhin, VKalVrt - A package for vertex reconstruction in ATLAS, ATL-PHYS-2003-031 (2003).

[5] The ATLAS Collaboration, Expected Performance of the ATLAS Experiment, Detector, Trigger and Physics, CERN-OPEN-2008-020, Geneva, 2008.

[6] P. Avery, http://www.phys.ufl.edu/ avery/fitting.html.

[7] G. Piacquadio, C. Weiser, A new inclusive secondary vertex algorithm for b-jet tagging in ATLAS, CHEP 2007, Journal of Physics: Conference Series 119 (2008) 032032. 